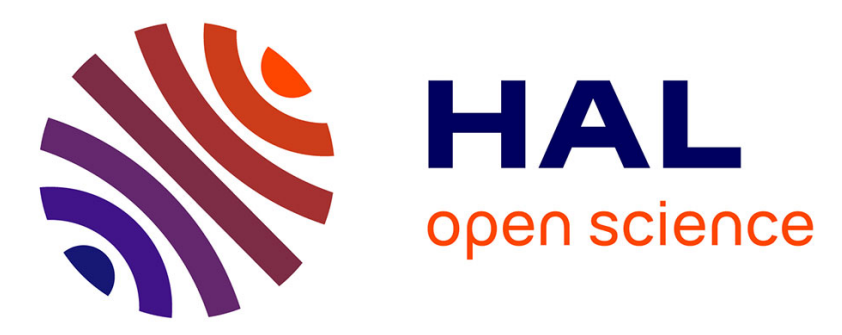

\title{
Force Sensing with an Optomechanical Self-Oscillator
} Biswarup Guha, Pierre Etienne Allain, Aristide Lemaitre, Giuseppe Leo, Ivan

Favero

\section{To cite this version:}

Biswarup Guha, Pierre Etienne Allain, Aristide Lemaitre, Giuseppe Leo, Ivan Favero. Force Sensing with an Optomechanical Self-Oscillator. Physical Review Applied, 2020, 14 (2), 10.1103/PhysRevApplied.14.024079 . hal-03052627

\section{HAL Id: hal-03052627 \\ https://hal.science/hal-03052627}

Submitted on 10 Dec 2020

HAL is a multi-disciplinary open access archive for the deposit and dissemination of scientific research documents, whether they are published or not. The documents may come from teaching and research institutions in France or abroad, or from public or private research centers.
L'archive ouverte pluridisciplinaire HAL, est destinée au dépôt et à la diffusion de documents scientifiques de niveau recherche, publiés ou non, émanant des établissements d'enseignement et de recherche français ou étrangers, des laboratoires publics ou privés. 


\title{
Force Sensing with an Optomechanical Self-Oscillator
}

\author{
Biswarup Guha $\odot,{ }^{1}$ Pierre Etienne Allain, ${ }^{1}$ Aristide Lemaitre, ${ }^{2}$ Giuseppe Leo,${ }^{1}$ and Ivan Favero ${ }^{1, *}$ \\ ${ }^{1}$ Matériaux et Phénomènes Quantiques, Université de Paris, CNRS UMR 7162, 10 rue Alice Domon et Léonie \\ Duquet, Paris 75013, France \\ ${ }^{2}$ Centre de Nanosciences et de Nanotechnologies, CNRS, Université Paris-Saclay, Palaiseau 91120, France
}

(Received 19 May 2018; revised 13 May 2020; accepted 24 June 2020; published 26 August 2020)

\begin{abstract}
Ultra-high-frequency nanomechanical resonators $\left(f_{m}>300 \mathrm{MHz}\right)$ can increase our capacity to study fast physical phenomena, for example by measuring forces. Their extreme stiffness is also a chance to access molecular forces in the subpicometer low amplitude of motion limit, but it makes them hard to drive and control. Here we analyze a method to optomechanically sense a force field with an ultrahigh-frequency and stiff mechanical resonator, where back-action optical forces set the resonator into a self-sustained stable oscillator trajectory. After elucidating the experimental conditions to obtain optimal resolution, we carry out controlled experiments where the oscillator senses an optical force generated by a secondary laser. We analyze and model our results, and illustrate the concrete advantage of the method in the measurement of such a weak force, which would otherwise remain undetected by the undriven probe. We establish the thermodynamical limits of the approach, and finally connect it to the class of feedback-controlled problems, clarifying its assets and limitations.
\end{abstract}

DOI: 10.1103/PhysRevApplied.14.024079

\section{INTRODUCTION}

Micro and nanoelectromechanical systems (NEMS) have been used as mass sensors for biological and chemical applications [1-4], where their miniature dimensions enable sensitive measurements [5-9]. Thanks to their high frequencies up to the GHz range, NEMS also also bring the promise of high-speed operation, beyond that reached by conventional force or acceleration sensors, and may allow tracking short time-scale physical interactions. Highfrequency mechanical resonators are often stiff, hence their Brownian motion is also small, down to a hundred of femtometers for a $\mathrm{GHz}$ resonator of picogram mass at room temperature, which is the perfect scale to measure molecular forces in the low-amplitude nonperturbative limit. Despite these combined merits, the promising applications of high-frequency stiff NEMS are hindered by the fact that their motion is difficult to drive and measure with ultimate signal to noise using established electrical methods. Recently, nanomechanical systems have been coupled to the light confined in an optical cavity, in a plethora of geometries designed for quantum-physics research [10, 11]. While the resulting optomechanical devices were recognized early to enable broadband optical detection of motion [12], their use in sensing technologies is still under development [13-16]. New optomechanical sensing

\footnotetext{
*ivan.favero@u-paris.fr
}

methods are yet to be unveiled that would bring added functionality and/or performance.

Here we investigate how dynamical optomechanical effects can facilitate and improve the use of high-frequency stiff NEMS probes in mechanical sensing. Our experiments are carried out on ultra-high-frequency $\left(300 \mathrm{MHz}<f_{m}<\right.$ $3 \mathrm{GHz}$ ) mechanical resonators with a modal stiffness of $k \sim 10^{5} \mathrm{~N} / \mathrm{m}$. By dynamically coupling such a resonator to a laser-pumped optical cavity, we generate an oscillatory mechanical trajectory and observe the trajectory's perturbation under an applied force. We examine the conditions to obtain the most stable trajectory, hence the best force resolution, and use a second laser to apply a weak optical force. We show that the optically dressed probe can detect the applied force. We explore the sources of noise affecting this optomechanical self-oscillation force-sensing method, show that it can approach the thermodynamical limit of detection, and elucidate its assets and limitations. The technique appears as a valuable tool for the measurement of weak forces by ultra-high-frequency NEMS probes operating in the low amplitude of motion limit. It works irrespective of the nature of the force, conservative or dissipative, and may hence be applied to a large variety of physical interactions.

\section{PRINCIPLE}

The principle of optomechanical self-oscillation force sensing is sketched in the frequency domain in the panels 


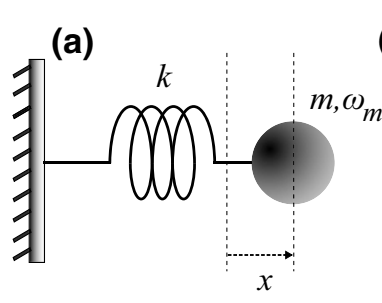

(b)

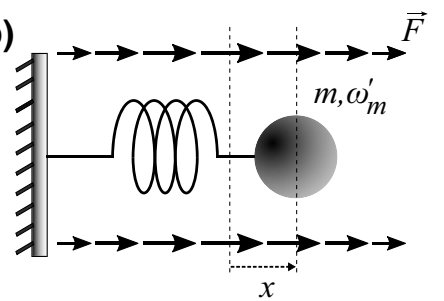

(c)

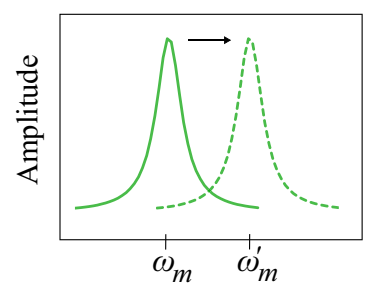

(d)

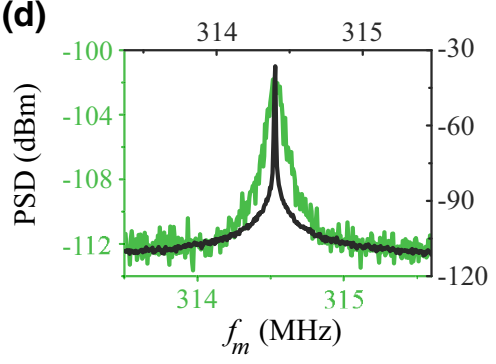

FIG. 1. (a) A mass on a spring mechanical resonator has a resonance frequency $\omega_{m}$. (b) Once placed in a force field, the resonator experiences to first order a linear spatial variation of the force. (c) As a consequence, the resonance frequency is shifted to $\omega_{m}^{\prime}$ by the gradient of the force. (d) Mechanical spectra of a GaAs disk with frequency of the first-order $\operatorname{RBM} f_{m}=\omega_{m} / 2 \pi \sim$ $300 \mathrm{MHz}$. The green curve is measured in the Brownian motion regime, while the black curve is in the regime of optomechanical self-oscillation, showing strong line narrowing, and hence capacity to resolve a frequency shift.

of Fig. 1. A mass $m$ on a spring $k$ [Fig. 1(a)] has a resonant mechanical frequency $\omega_{m}^{2}=k / m$ and vibrates with a small amplitude $x$ around an equilibrium position $x_{0}$. Once positioned within a force field $F(x)$ [Fig. 1(b)], it experiences to first order a force $F\left(x_{0}\right)+\nabla F \cdot x$ and its frequency is hence shifted to $\omega_{m}^{\prime}=(k-\nabla F / m)^{1 / 2}$. The frequency shift $\sim \omega_{m}(\nabla F / 2 k)$, taken as a force gradient estimator, is easily detected when superior to the spectral line width of the mechanical signal [Fig. 1(c)]. It can be determined with high resolution by harmonically forcing and tracking the resonator's response by electrical means, which is the most standard approach in resonant mechanical sensing and atomic force microscopy (AFM). In the so-called slope-detection method [17], the frequency of the drive is tuned to a flank of the mechanical resonance, such that small frequency shifts are transduced in amplitude variations. Since electromechanical forcing and tracking of ultra-high-frequency $\left(f_{m}>300 \mathrm{MHz}\right)$ and stiff NEMS probes are difficult to implement with an optimal signal-to-noise ratio set by the thermomechanical limit, we explore here another strategy. We employ an optical approach and make use of dynamical couplings in order to reduce the line width of the mechanical signal and facilitate force detection. This idea turns out to be efficiently implemented using optomechanical effects: above a certain threshold of optomechanical interaction, the gain provided by back-action optical forces acting on the mechanical system surpasses losses and the motion becomes highly coherent and harmonic [18-24]. In the frequency domain, the mechanical line narrows abruptly, resembling a lasing transition [18,21]. Because this oscillation regime relies on an intrinsic optomechanical feedback effect, the sensing method explored here can be perceived as an all-optical analog of the frequency modulation technique introduced in AFM [25], albeit operating in the ultra-high frequency range and with no external feedback loop required. Figure 1(d) shows an experimental example of optomechanical line narrowing obtained on the ultra-high-frequency and stiff mechanical probe employed in this work. Below, we explore the optimal conditions to employ this optomechanical self-oscillator in force-sensing applications, and use it to resolve a weak optical force of dissipative nature. We finally clarify the assets and limitations of this method.

\section{OPTOMECHANICAL DISK SELF-OSCILLATORS}

Our experiments are carried out on semiconductor optomechanical disk resonators, which couple light stored in high- $Q$ optical whispering gallery modes (WGMs) to the mechanical radial breathing modes (RBMs) of the structure [26-28]. These disks are fabricated out of an epitaxial wafer of (aluminium) gallium arsenide $(\mathrm{Al}, \mathrm{Ga}) \mathrm{As}$ with the following composition: $\mathrm{GaAs}(200 \mathrm{~nm}) / \mathrm{Al}_{0.8} \mathrm{Ga}_{0.2} \mathrm{As}(1800$ $\mathrm{nm}) / \mathrm{GaAs}$ (substrate). We use electron-beam lithography and subsequent wet etching to pattern and fabricate the disk resonators, which are then elevated over the substrate on a mesa structure in order to be optically addressed by fiber-taper evanescent coupling techniques [24,28,29]. Figure 2(a) shows a GaAs disk atop an (Al, Ga)As pedestal positioned over a mesa. Below, without further mention, the GaAs disk we employ has a radius of $4.5 \mu \mathrm{m}$ and

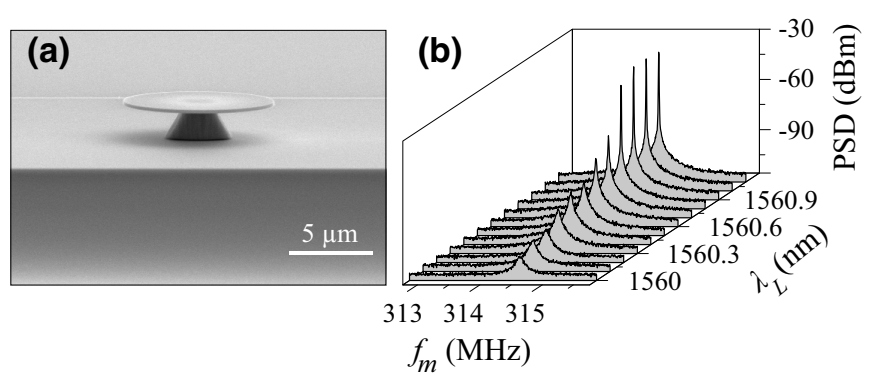

FIG. 2. (a) A GaAs disk atop an (Al, Ga)As pedestal atop a mesa. The disk radius is $4.5 \mu \mathrm{m}$ and the thickness $200 \mathrm{~nm}$. (b) Evolution of the rf mechanical spectrum associated to the first order RBM of the disk, as a function of the pump laser wavelength $\lambda_{L}$. The laser is tuned to the blue flank of the WGM optical resonance, and the detuning progressively reduced as the pump wavelength is increased. The power incident on the cavity is of $108 \mu \mathrm{W}$. 
a thickness of $200 \mathrm{~nm}$. Let us first examine the conditions to transform such a resonator into an optomechanical self-oscillator.

Figure 2(b) shows a series of rf spectra of the output light of the optomechanical system, around the frequency of the first-order RBM. The spectra are acquired in ambient conditions, as the pump laser is blue detuned on the flank of an optical WGM resonance and its wavelength progressively increased to reduce the detuning. As the detuning is decreased, the number of photons injected into the resonator increases, which intensifies optomechanical effects. Under these conditions, the mechanical RBM motion is progressively amplified along the series of curves, up to the point where the amplification overcomes mechanical losses and the spectral resonance abruptly narrows. At this point, the motion is self-sustained into an harmonic trajectory (see phase-space representations in Appendix A).

We explore first the optomechanical interactions responsible for this dynamical behavior. The light trapped within a WGM excites the RBM motion through three optomechanical mechanisms: radiation pressure, which pushes the disk sidewalls apart; electrostriction, which stresses the resonator's constitutive material outwards; and the photothermal force, which is a consequence of light absorption and thermal expansion of the disk structure. The three forces act on the RBM in the same direction, but the first two are conservative while the third is dissipative in nature. The full dynamics of the system can be modeled by a generalized set of coupled equations that include these three mechanisms [24]:

$$
\begin{aligned}
\dot{a}= & -\frac{\kappa}{2} a \\
& +i\left[\Delta \omega+g_{\mathrm{om}} x+\frac{\omega_{0}}{n} \frac{d n}{d T} \Delta T\right] a \\
& +\sqrt{\kappa_{\mathrm{ex}}} a_{\mathrm{in}} \\
m \ddot{x}+m \Gamma_{m} \dot{x}+m \omega_{m}^{2} x= & F_{\mathrm{opt}}+F_{\mathrm{pth}}+F_{L} \\
\dot{\Delta T}= & -\frac{\Delta T}{\tau_{\mathrm{th}}}+\frac{\Gamma_{\mathrm{pth}}|a|^{2}}{\tau_{\text {th }}}
\end{aligned}
$$

with $a$ the cavity field normalized such that $|a|^{2}$ is the number of intracavity photons, $\kappa$ the optical cavity decay rate, $\Delta \omega=\omega_{L}-\omega_{0}$ the laser-cavity detuning, $\omega_{L}=$ $2 \pi c / \lambda_{L}\left(\omega_{0}\right)$ the laser (cavity) frequency, $g_{\text {om }}=-\frac{\partial \omega_{0}}{\partial x}$ the frequency-pull parameter, $n$ the refractive index, $d n / d T$ the thermorefractive coefficient, $\Delta T$ the temperature increase within the disk and $\kappa_{\mathrm{ex}}$ the coupling rate of the cavity to the optical input field $a_{\mathrm{in}}$, normalized such that $\left|a_{\mathrm{in}}\right|^{2}=P_{L}$ is the input laser power. In the second line $F_{\mathrm{opt}}=F_{\mathrm{RP}}+F_{\mathrm{el}}$ is the sum of conservative optical forces (radiation pressure and electrostriction), while $F_{\mathrm{pth}}$ is the dissipative photothermal force and $F_{L}$ the Langevin force associated to
TABLE I. Radiation pressure, electrostriction, and photothemal force exerted onto the RBM by a single photon trapped within the considered WGM, along with the thermal relaxation time of the disk. All values are for the specific GaAs disk considered in this work, operated under ambient conditions.

\begin{tabular}{lccc}
\hline \hline$F_{\mathrm{RP}}^{1}(\mathrm{~N})$ & $F_{\mathrm{el}}^{1}(\mathrm{~N})$ & $F_{\mathrm{pth}}^{1}(\mathrm{~N})$ & $\tau_{\mathrm{th}}(\mu \mathrm{s})$ \\
\hline $1.45 \times 10^{-14}$ & $2.94 \times 10^{-14}$ & $6.48 \times 10^{-9}$ & 0.20 \\
\hline \hline
\end{tabular}

the mechanical damping rate $\Gamma_{m}$. In the third equation, $\tau_{\text {th }}$ is the thermal relaxation time of the resonator and $\Gamma_{\mathrm{pth}}=$ $R_{\mathrm{th}} \kappa_{\mathrm{abs}} \hbar \omega_{L}$, with $\kappa_{\mathrm{abs}}$ the intracavity absorption rate and $R_{\mathrm{th}}$ the thermal resistance of the disk that links the temperature increase to the intracavity absorbed power $\kappa_{\mathrm{abs}} \hbar \omega_{L}|a|^{2}$, such that in the steady state $\Delta T=\Gamma_{\text {pth }}|a|^{2}$.

The set of equations (1) allows full modeling of the optically induced amplification of mechanical motion, provided the involved model parameters are known. $\omega_{0}, \kappa, \kappa_{\mathrm{ex}}$, $\omega_{m}, m$, and $\Gamma_{m}$ are measured through a linear spectroscopy of the resonator [26]. The data reported in Fig. 2(b) are obtained with a WGM of radial order $p=5$, whose coupling $g_{\text {om }}$ to the first-order RBM of the disk is calculated by finite-element-method (FEM) simulations [28,30], allowing determination of the force per photon associated to radiation pressure $\left(F_{\mathrm{RP}}^{1}\right)$ and electrostriction $\left(F_{\mathrm{el}}^{1}\right)$ through the relations $F_{\mathrm{RP}}^{1}=\hbar g_{\mathrm{om}}^{\mathrm{geo}}$ (geometric coupling) and $F_{\mathrm{el}}^{1}=$ $\hbar g_{\mathrm{om}}^{\mathrm{PE}}$ (photoelastic coupling) [30]. These forces are listed in Table I along with the photothermal force per photon $F_{\text {pth }}^{1}$, which is evaluated from the known thermoelastic properties of the resonator and from the parameter $\Gamma_{\mathrm{pth}}$. The latter is independently measured by fitting the thermooptic response of the resonator at large optical power [24,31-33]. Finally $\tau_{\text {th }}$ is obtained from a measurement of the frequency response of our GaAs disk resonator to a rf modulation of the input light (see Appendix B), closing the set of parameters required for the model. Finally, all optical forces at play if our system are known independently, as well the mechanical response of the device to them. This enables a full modeling and analysis of our experiments.

The values reported in Table I show that, under ambient conditions, the employed RBM mode of the disk experiences an important contribution from the photothermal force. In the dynamical back-action regime however, the response time of the force matters on top of its mere static force amplitude [34]. The complete dynamical analysis actually shows that the three types of interactions (radiation pressure, electrostriction, and photothermal) participate to the amplification of mechanical motion reported in Fig. 2(b), finally leading to the self-oscillation trajectory (see an equivalent analysis detailed in Ref. [24]). The exact nature of the forces triggering the self-oscillation may not be crucial per se, but is of interest below, where a controlled optical force is generated by a second laser and sensed by the oscillator. 


\section{PHASE NOISE OF THE OPTOMECHANICAL SELF-OSCILLATOR}

Just like for an electronic oscillator, the performances of an optomechanical self-oscillator can be characterized by its phase noise and frequency stability. These parameters are crucial in the proposed force-sensing protocol, as they set the resolution in force gradient when the oscillator's frequency is taken as the estimator. In the absence of optomechanical effects, the mechanical resonance line width $\left(\delta f_{m}=\Gamma_{m} / 2 \pi\right)$ is determined by geometrical and structural properties of the disk resonator, along with its interaction with a possible surrounding fluid [16,35-38]. In the self-oscillation regime however, the oscillator line width is also determined by the operation conditions and by the noise sources at play in the system or in its input. Drawing on an analogy with lasers, the line width of optomechanical self-oscillators has been described using a general theory of "line narrowing" in self-sustained oscillators [39], proposing an expression for the "SchawlowTownes line width" [40] of an oscillator merely affected by thermomechanical noise:

$$
\delta f_{m, \mathrm{osc}}=\frac{\pi k_{B} T}{P}\left(\delta f_{m}\right)^{2},
$$

where $T$ is the bath temperature and $P$, the oscillator output power, which can be written in terms of the energy stored in the resonator $P=\Gamma_{m} E_{\text {stored }}$ such that

$$
\delta f_{m, \text { osc }}=\frac{k_{B} T}{2 E_{\text {stored }}} \delta f_{m} .
$$

Equation (3) indicates that the oscillator's line width scales inversely with the stored energy, pointing towards the advantage of an energetic oscillator for high resolution. That said, Eqs. (2) and (3) rely on the assumption of a thermomechanical "white noise" and neglect other possible noise sources [41]. It is hence worth measuring the phasenoise spectrum of our optomechanical oscillator to better clarify optimal conditions for force-sensing applications.

Figure 3(a) shows a phase-noise spectrum measured on our GaAs optomechanical disk of radius $4.5 \mu \mathrm{m}$ and thickness $200 \mathrm{~nm}$, self-oscillating on its first order RBM at a frequency $f_{m} \simeq 314 \mathrm{MHz}$. For carrier-frequency offsets between $10^{4}$ and $10^{6} \mathrm{~Hz}$, the phase-noise spectral density varies as $1 / f^{2}$ (dashed orange line), which is the signature of a white-noise source [41]. However, spectral bumps appear between $10^{2}$ and $10^{4} \mathrm{~Hz}$, which are observed to disappear when the disk is evanescently coupled to a rigid on-chip waveguide (see Fig. 8 of Appendix C). They are associated to fiber taper coupling instabilities in our setup. As the stored energy $E_{\text {stored }}$ increases with the drive optical power, we measure in Fig. 3(b) the phase-noise spectra for the same oscillator as in Fig. 3(a), but for varying input power. We observe that the phase noise reduces as power
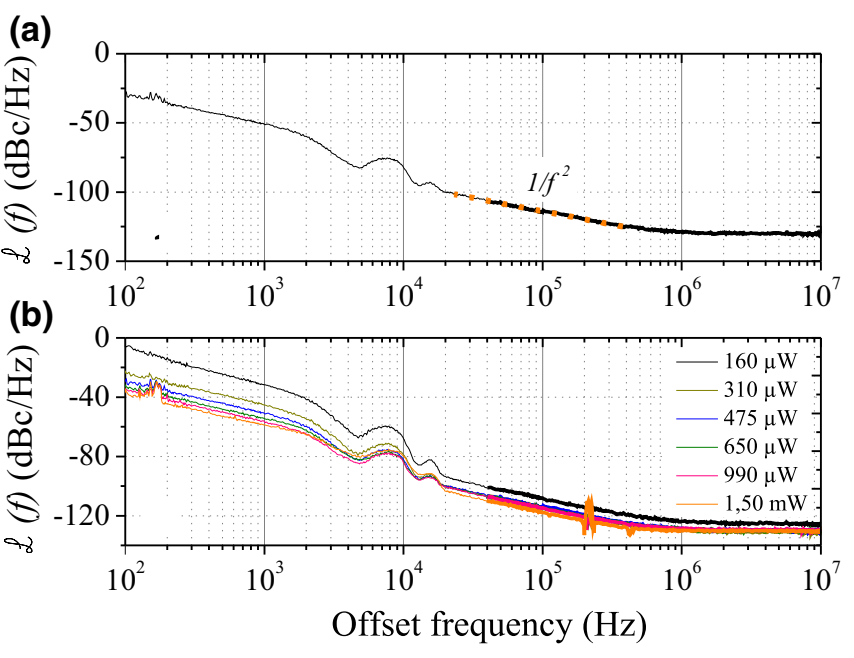

FIG. 3. (a) Phase-noise spectrum of a GaAs disk optomechanical self-oscillator. The dashed orange line indicates a $1 / f^{2}$ dependence of the phase-noise spectral density. The measurements are obtained just above threshold at $\lambda_{L}=1561 \mathrm{~nm}$ with an optical power of $475 \mu \mathrm{W}$ traveling in the fiber taper and incident on the cavity. (b) Phase-noise spectra of the same GaAs disk self-oscillator for varying drive optical power impinging on the cavity.

increases, as expected from Eq. (3) and observed on other systems $[39,42]$. This underlines the interest of operating disk optomechanical oscillators at large optical power for high-resolution sensing. At the same time, a too large optical drive results in nonlinearities in the mechanical to optical transduction, limiting the range of measurements (see Appendix A). A compromise needs finally to be chosen to reach optimal conditions, as described in the following section.

\section{FORCE SENSING WITH AN OPTOMECHANICAL DISK SELF-OSCILLATOR}

We now implement the proposed principle of selfoscillation force detection with the ultra-high-frequency and stiff optomechanical disk probe, using the experimental setting shown in Fig. 4(a). A first laser of wavelength $\lambda_{1}$ is used to inject light in a first WGM of the disk (WGM 1 resonating at $\lambda \sim 1532 \mathrm{~nm}$ ), in order to drive the resonator into optomechanical self-oscillation like reported in the previous sections. Once the self-oscillation regime is reached and the optimal drive conditions are selected (see above), we apply a test force onto the oscillator. We chose to apply an optical force, because the full and independent optomechanical characterization of the disk carried above enables its complete analysis and modeling. A second laser of wavelength $\lambda_{2}$ is hence used to inject light into a second WGM of the disk (WGM 2 resonating at $\lambda \sim 1590$ $\mathrm{nm}$ ), in order to create a weak optical force acting on the 

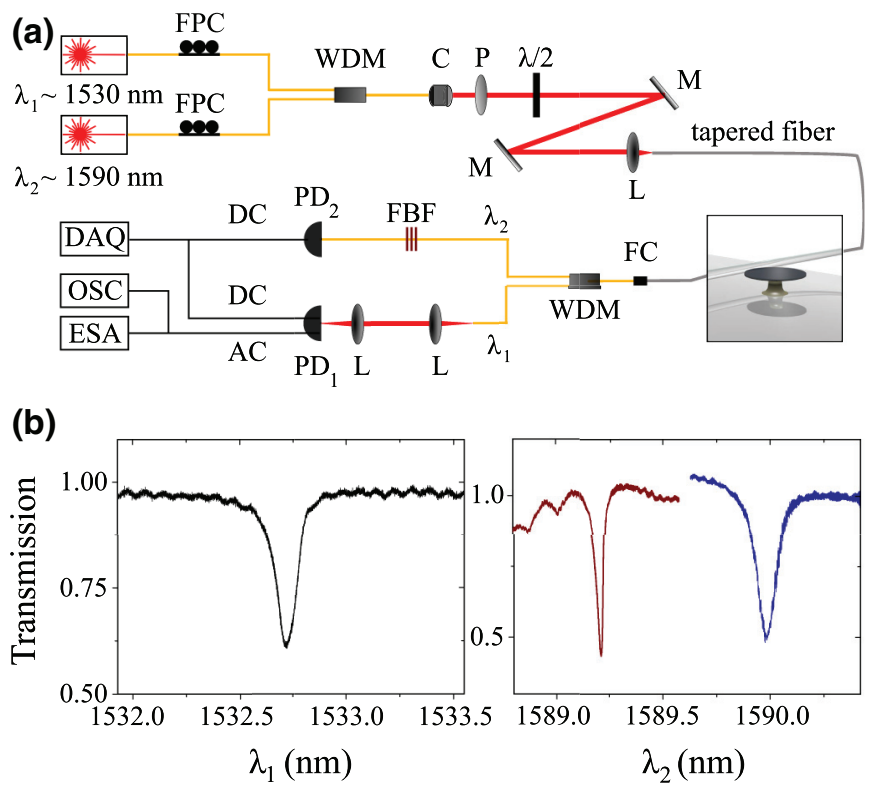

FIG. 4. (a) Experimental setup for force-sensing experiment with a GaAs disk optomechanical self-oscillator. FPC, fiber polarization controller; WDM, wavelength division multiplexer; $\mathrm{C}$, collimator; $\mathrm{P}$, linear polarizer; $\lambda / 2$, half-wave plate; $\mathrm{M}$, mirrors; L, lenses; FBF, fibered Bragg filter; PD1 and PD2, photodiodes; DAQ, data acquisition card; OSC, oscilloscope; ESA, electronic spectrum analyzer; FC, fiber connector. The yellow lines indicate commercial optical fibers, the gray bare fibers, the red free-space beams, and the black electric cables. (b) Left panel: optical spectrum of WGM1 when the disk is at rest. Right panel: optical spectra of WGM 2, measured by the second laser when the disk is at rest with the first laser switched off (maroon) and when the disk is optically driven by the first laser just above self-oscillation threshold (blue).

oscillating RBM. By tuning $\lambda_{2}$ to the flank of the resonance of WGM 2, we generate a gradient for this optical force, which is then detected by tracking the oscillator's frequency. The second laser is operated at much smaller power than the first (in practice $P_{1} / P_{2} \sim 40$ ), which guarantees that the optomechanical self-oscillation conditions are not disrupted when applying the force. The two laser fields propagate through the same fiber taper to reach the disk resonator, but are then split at the output by a wavelength division multiplexer device. The strong field of laser 1 is filtered out from the output channel 2 by a fiber Bragg filter, in order to obtain a precise read-out of the second laser field alone.

The two employed WGMs, whose spectrum is visible in Fig. 4(b), are TE polarized of radial order $p=5$ but possess a distinct azimuthal number $m-1$ and $m+1$ [43]. The left panel shows the resonant optical spectrum of WGM 1, acquired by scanning the first laser's wavelength when the disk is at rest (black curve). The right panel shows the resonant optical spectrum of WGM 2, acquired by scanning the second laser's wavelength when the disk

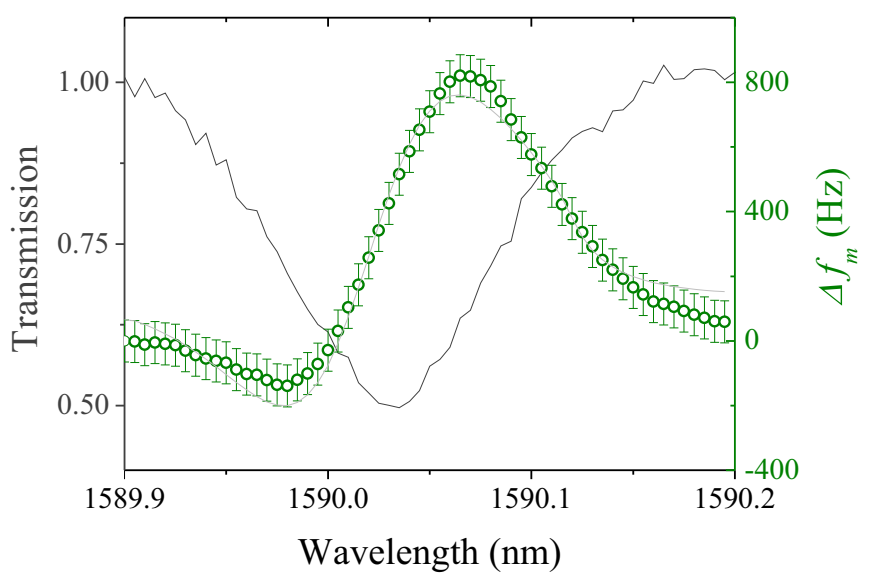

FIG. 5. Optical force sensing with a disk optomechanical self-oscillator. The mechanical frequency shift $\Delta f_{m}$ of the selfoscillator driven by the first laser (green open circles) is shown as a function of the wavelength of the second laser (see text). The gray line is a fit by our model. The black curve represents the optical fiber taper transmission close to the resonance of WGM 2 , acquired by time integrating the optical intensity in the second output channel when the disk is self-oscillating.

is at rest (maroon curve) and when the disk is set in selfoscillation by the first laser (blue curve). When the disk self-oscillates there is a thermo-optic redshift of WGM2 due to heating of the disk by laser 1 , and we also observe an increase of the apparent line width of WGM $2\left(\Delta \lambda^{\mathrm{SO}}=\right.$ $140 \mathrm{pm}$, to compare with $\Delta \lambda=40 \mathrm{pm}$ when the disk is at rest). This increase is a consequence of the finite amplitude of the self-oscillatory motion, which through optomechanical coupling enforces a spectral oscillation (at frequency $f_{m}$ ) of the resonating wavelength of WGM2, and hence a broadening in the slow spectroscopy reported in Fig. 4(b). In order to limit this effect and avoid a complete distortion of the WGM2 resonance, the disk is operated just above the self-oscillation threshold in subsequent experiments.

Under these conditions, the force-sensing experiment is carried out by scanning $\lambda_{2}$ over the resonance of WGM2 while tracking the self-oscillator frequency. Varying this way the detuning between the second laser and WGM2, we vary the gradient of the weak optical force applied onto the RBM and test in detail our sensing protocol, as well as our understanding of the experiment. Figure 5 shows the experimental results, with the self-oscillator's frequency shift $\Delta f_{m}=f_{m}^{\prime}-f_{m}$ (green open circles) plotted together with the optical fiber taper transmission close to the resonance of WGM 2 (black curve), for reference, both being acquired as function of $\lambda_{2}$, after integration on a time scale $\gg\left(1 / \omega_{m}, \tau_{\mathrm{th}}\right)$. We observe that the frequency shift varies with $\lambda_{2}$, as expected, and attains a maximal value of $800 \mathrm{~Hz}$ (relative shift $2.6 \times 10^{-6}$ ). This corresponds to an applied force gradient of $1.5 \mathrm{~N} / \mathrm{m}$, which is easily detected despite the extreme stiffness of the probe $k \sim 10^{5} \mathrm{~N} / \mathrm{m}$. 
The nature and amplitude of the force being sensed can be analyzed using the dynamical model we introduce above, which includes radiation pressure, electrostriction, and photothermal forces [Eq. (1)]. This time the model is applied to WGM2 instead of WGM1, and it is linearized around an equilibrium point [24], leading to the following expression for the shifted mechanical frequency $\omega_{m}^{\prime}$ :

$$
\begin{aligned}
\omega_{m}^{\prime}= & \omega_{m}\left[1-\frac{|<a>|^{2} \hbar g_{\mathrm{om}}^{2}}{2 m \omega_{m}^{2}}\left\{\frac{\omega_{m}-\Delta \omega}{\left(\Delta \omega-\omega_{m}\right)^{2}+\frac{\kappa^{2}}{4}}\right.\right. \\
& \left.-\frac{\Delta \omega+\omega_{m}}{\left(\Delta \omega+\omega_{m}\right)^{2}+\frac{\kappa^{2}}{4}}\right\}-\frac{|<a>|^{2} g_{\mathrm{om}}}{2 m \omega_{m}^{2}} \frac{F_{\mathrm{pth}}^{1}}{1+\omega_{m}^{2} \tau_{\mathrm{th}}^{2}} \\
& \left.\left\{\frac{\omega_{m}+\omega_{m} \tau_{\mathrm{th}} \frac{\kappa}{2}-\Delta \omega}{\left(\Delta \omega-\omega_{m}\right)^{2}+\frac{\kappa^{2}}{4}}-\frac{\Delta \omega+\omega_{m}+\omega_{m} \tau_{\mathrm{th}} \frac{\kappa}{2}}{\left(\Delta \omega+\omega_{m}\right)^{2}+\frac{\kappa^{2}}{4}}\right\}\right],
\end{aligned}
$$

where $|\langle a\rangle|^{2}$ is the number of intracavity photons at equilibrium (in WGM2), $g_{\mathrm{om}}=g_{\mathrm{om}}^{\mathrm{geo}}+g_{\mathrm{om}}^{\mathrm{PE}}, \Delta \omega=\Delta^{b} \omega+$ $g_{\text {om }} x_{\text {eq }}+\left(\omega_{2} / n\right)(d n / d t) \Delta T_{\text {eq }}$ with $x_{\text {eq }}\left(\Delta T_{\text {eq }}\right)$ the mean displacement (temperature increase) associated to the equilibrium point, and $\Delta^{b} \omega=\omega_{L}-\omega_{2}$ the detuning to the resonance of the bare WGM2. As discussed above in the analysis of the self-oscillator and elsewhere [24], the parameters entering this expression are all evaluated in an independent manner, which enables fitting experimental results with in principle no free parameter. In Fig. 5, the gray line is the result of such a fit, where the parameters $\Gamma_{\text {th }}$ and $\tau_{\text {th }}$ are let to vary close to their first estimated value in order to account for the residual uncertainty in the resonator pedestal's thermal properties and dimensions (inferred in the SEM). The parameters entering this linearized model are listed in Table II, and lead to a good agreement with experiments. Note the asymmetry of the frequency shift as a function of $\lambda_{2}$, which results from the thermo-optic effect, as detailed in Appendix D. As already discussed in Table I, the optical force exerted by photons stored in the WGM, and sensed here by the oscillator, is dominantly a dissipative photothermal force, with small associated radiation pressure and electrostriction components.

Our results involve measured frequency shifts $\Delta f_{m}$ of hundreds of Hz. The RBM we employ having a natural mechanical line width $\delta f_{m}$ of $100 \mathrm{kHz}\left(Q_{m}\right.$ of $\left.\sim 3000\right)$, such frequency shifts would have been impossible to resolve in the Brownian motion regime. In the experiments reported in Fig. 5, the minimum detectable shift is shown as an error bar of the green open circles and amounts to $\Delta f_{m}=130 \mathrm{~Hz}$, which corresponds to a relative shift $\Delta f_{m} / f_{m}$ of $4 \times 10^{-7}$. This corresponds to a detectable force gradient of $0.9 \times 10^{-1} \mathrm{~N} / \mathrm{m}$, out of reach of our extremely stiff probe when operated in the thermal motion regime
TABLE II. Parameters used in the force-sensing model.

\begin{tabular}{lc}
\hline \hline Parameter & Measured value \\
\hline$m$ & $67.6 \mathrm{pg}$ \\
$\kappa_{\text {in }, 1}$ & $6.055 \times 10^{10} \mathrm{rad} / \mathrm{s}$ \\
$\kappa_{\text {ex }, 1}$ & $7.705 \times 10^{9} \mathrm{rad} / \mathrm{s}$ \\
$P_{L, 1}$ & $1.04 \mathrm{~mW}$ \\
$\kappa_{\text {in }, 2}$ & $1.176 \times 10^{11} \mathrm{rad} / \mathrm{s}$ \\
$\kappa_{\text {ex }, 2}$ & $3.684 \times 10^{10} \mathrm{rad} / \mathrm{s}$ \\
$P_{L, 2}$ & $2.94 \mu \mathrm{W}$ \\
$\Gamma_{\text {pth }}$ & $2.374 \times 10^{-4} \mathrm{~K} / \mathrm{photon}$ \\
$\tau_{\text {th }}$ & $0.1 \mu \mathrm{s}($ fit $)($ measured $0.2 \mu \mathrm{s})$ \\
\hline \hline
\end{tabular}

(see Appendix D and Fig. 9 for the full force-sensing curve expressed in force gradient).

This detection capability demonstrates the practical advantage brought by the self-oscillation approach for force sensing: it provides us with a high-resolution below the ppm, but at the same time does not require to sinusoidally force the mechanical motion to reach this performance. Such forcing would indeed be challenging to achieve with satisfying signal-to-noise ratio using electromechanical methods, given the ultra-high-frequency range at play $\left(300 \mathrm{MHz} \ll f_{m}<3 \mathrm{GHz}\right.$ ) and the associated stiffness. Sinusoidal forcing and detection of surface acoustic waves in the few hundreds of $\mathrm{MHz}$ range was, for example, used for gas sensing, but with a signal-to-noise ratio that did not reach the thermodynamical limit $[44,45]$. Optomechanical self-oscillation force sensing seems to provide here a convenient solution. In the next section, we clarify how far this sensing method could be further developed.

\section{RESOLUTION OF FORCE SENSING WITH OPTOMECHANICAL SELF-OSCILLATION}

In this last section, we clarify the ultimate resolution capabilities of the optomechanical self-oscillation forcesensing method. We consider the ideal case where the self-oscillator's noise is solely governed by a white-noise source, leading to the following power spectral density of phase noise [41]:

$$
\mathscr{L}(f)=\frac{1}{\pi} \frac{\delta f_{\text {osc }}}{\delta f_{\text {osc }}^{2}+f^{2}},
$$

with $\delta f_{\text {osc }}$ the line width of oscillation and $f$ the frequency offset from the carrier. In the limit $f \gg \delta f_{\text {osc }}$, Eq. (5) is approximated as

$$
\mathscr{L}(f) \simeq \frac{1}{\pi} \frac{\delta f_{\text {osc }}}{f^{2}}
$$


In the power-law expansion of the phase-noise spectrum, the white-noise frequency component is given by [41]

$$
\mathscr{L}(f)=\frac{1}{2} b_{-2} f^{-2},
$$

which together with Eqs. (6) and (3) leads to the relation

$$
b_{-2}=\frac{1}{\pi} \frac{k_{B} T}{E_{\text {stored }}} \delta f_{m} .
$$

Knowing that the frequency jitter of an oscillator affected by a white-noise source is characterized by an Allan variance given by [41]

$$
\sigma_{f_{m}}^{2}(\tau)=\frac{b_{-2}}{2 \tau} .
$$

We finally obtain the minimum resolvable frequency shift of an ideal optomechanical self-oscillator:

$$
\frac{\sigma_{\omega_{m}}(\tau)}{\omega_{m}}=\sqrt{\frac{k_{B} T}{E_{\text {stored }}}} \sqrt{\frac{B}{2 \pi Q_{m} f_{m}}},
$$

where $B=1 / \tau$ is the measurement bandwidth and $\tau$ the acquisition time.

Equation (10) turns out to be exactly equal to the thermomechanical limit established for the slope-detection method, standardly employed in mechanical sensing at low frequency, where the mechanical resonator is resonantly driven by a sinusoidal force and its frequency tracked by monitoring the motion's amplitude or phase [17]. As the minimum detectable shift determines the resolvable force gradient, the outcome of our analysis is that the optomechanical self-oscillation force sensing method has the exact same thermodynamical force resolution as the slope-detection method. Our finding can actually be understood back from established results on feedback-controlled systems. The optomechanical dynamical back-action effect leading to optical cooling or amplification of motion can indeed be depicted as an intrinsic closed-loop feedback effect, where optical forces are linearly acting back on the mechanical resonator [10]. It is an established result that as long as the feedback is linear, there is in principle no gain in resolution to expect from a feedback-controlled mechanical device, as pointed out by independent works on optically cooled mechanical resonators [46,47]. Indeed, if the feedback cooling reduces the amount of fluctuations of the resonator, it reduces at the same time the resonator's susceptibility to external forces by the same amount, finally canceling any advantage. The optomechanical amplification regime, which just as cooling results from a linear feedback mechanism but with an opposite sign, cannot increase the ultimate force-gradient resolution either. As the present method employs the optomechanical self-oscillation regime, which lies beyond the mere regime of amplification, the question of a potential resolution increase must a priori be reconsidered. Our analysis indicates that as long as the amplitude of self-oscillation remains moderate the dynamics of the system can still be described by a linear optomechanical coupling, hence general results on linear feedbacks must hold true. Note finally that a similar conclusion was reached in the context of frequency modulation atomic force microscopy: $\mathrm{kHz}$ cantilevers set in oscillation using an external electronic feedback loop showed the same thermodynamical limit of force detection as cantilevers operated with the slope-detection technique [25].

\section{CONCLUSION}

In conclusion, we investigate in detail the idea of using an optomechanical self-oscillator as an efficient mechanical force sensor, clarifying the optimal conditions to reach performance. Even though the principles of operation completely differ from the conventional slope-detection technique, we establish the important result that the optomechanical self-oscillation method meets the same theoretical thermodynamical limit in resolution. However, by performing and modeling experiments on a concrete device, we demonstrate that the method leads in practice to an appreciable enhancement of the detection capability in situations where the slope-detection technique cannot be efficiently implemented to reach ultimate limits of detection. This is, in particular, relevant for ultra-high-frequency and very stiff mechanical resonators, which have the potential to resolve fast phenomena in the low-amplitude of motion regime. While very high frequency (approximately $\mathrm{MHz}$ ) electronanomechanical oscillators were already employed in sensing applications [48-50], they did not operate at the thermomechanical noise limit. In contrast, optomechanical detection has a quasi-infinite bandwidth and allows resolving thermomechanical fluctuations with a large signalto-noise ratio. This enables ultra-high-frequency optomechanical oscillators to approach the thermodynamical limit of force detection (see Appendix D), and may open a path to force-sensing bandwidths that approach the oscillation frequency $[51,52]$.

The optomechanical self-oscillation sensing technique brings additionally an advantage of compactness, as it requires a single continuous-wave laser and a single detector. It could hence be integrated in an autonomous optoelectronics architecture. Finally, it does run irrespective of the nature of the force being sensed, and can hence be applied to the investigation of dissipative forces. Such forces are of utmost importance in molecular physics and biology, because they provide information about conformation changes and their functional role: the possibility to track them at high speed constitutes a scientific challenge. Questions are still open regarding the extension of the optomechanical self-oscillation force-sensing method 
to nonlinear regimes, which are already touched at large optical power (see Appendix A), or to the quantum regime, where the noise merely results from vacuum fluctuations $[53,54]$. Optomechanical sensing approaches will certainly yet broaden their range of application, combining the precision of optical techniques with ultimately fast and low perturbative mechanical devices.

\section{ACKNOWLEDGMENTS}

The authors acknowledge support of the European Research Council via the GANOMS (306664) and NOMLI (770933) projects, of the European Commission via the VIRUSCAN (731868) project, and of the Agence Nationale de la Recherche through the Quantera QuaSeRT project.

\section{APPENDIX A: SELF-OSCILLATION IN PHASE SPACE}

In self-oscillation, the cavity resonance frequency $\omega_{0}$ is modulated in time by the large amplitude of mechanical motion, following the relation $\omega_{\text {cav }}(t)=\omega_{0}-g_{\text {om }} x(t)$. This results in a large temporal modulation of the cavity transmission $T(t)$, which follows the dynamics of the mechanical motion $x(t)$. Figures 6(a) and 6(b) show the temporal behavior of $T$ and the corresponding phase-space trajectory, when the detuning is such that the system sits just above the self-oscillation threshold. The 2D phasespace trajectory is obtained by considering the transmission $T$ and its time derivative $d T / d t$ as two conjugated degrees of freedom.
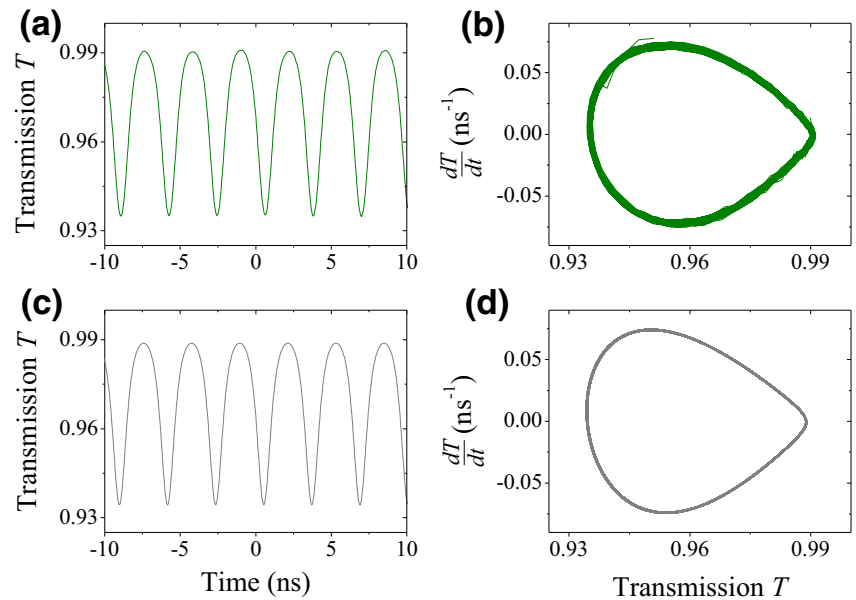

FIG. 6. Dynamics of optomechanical self-oscillation of a GaAs disk. (a) Measured normalized transmission $T$ as a function of time. (b) Phase-space representation of the measured dynamics. (c) Normalized transmission $T$ as a function of time from model. (d) Phase-space representation of the dynamics evaluated from the model.
To model this self-oscillation dynamics, we write the self-oscillation trajectory as a sinusoidal function:

$$
x(t)=A \cos \left(\omega_{m} t+\phi\right)
$$

and the time-varying transmission is evaluated using the relation:

$$
T(t)=\frac{[\Delta \omega(t)]^{2}+\left(\kappa_{\mathrm{in}} / 2-\kappa_{\mathrm{ext}} / 2\right)^{2}}{[\Delta \omega(t)]^{2}+\left(\kappa_{\mathrm{in}} / 2+\kappa_{\mathrm{ext}} / 2\right)^{2}} .
$$

The lower panels of Fig. 6 present the results of such modeling, which shows a good agreement with experimental results. The outcome of this analysis is that the nonlinearity of the mechanical-to-optical transduction, which is naturally embedded in Eq. (A2), is responsible for the distorted phase-space trajectories of Fig. 6 that depart from harmonic circles. This indicates that a nonlinear optical force feedback could already be at play in some self-oscillations trajectories, with implications for force sensing that are to be investigated.

\section{APPENDIX B: THERMAL RELAXATION TIME $\boldsymbol{\tau}_{\text {th }}$}

The thermal relaxation time $\tau_{\text {th }}$ is directly inferred from a measurement of the frequency response of the GaAs disk under illumination. To that purpose, the intensity of the laser light injected into the disk is modulated and the coherent amplitude response of the system monitored using a network analyzer plugged onto the output photodetector. Figure 7 shows the normalized response at low frequency, which is governed by the thermo-optic effect and hence reveals the thermal dynamics. $\tau_{\text {th }}$ is obtained by fitting the response function by $A+B /\left(1+i \omega \tau_{\mathrm{th}}\right)$, where $A$ and $B$ are real numbers and $\omega$ is the modulation frequency. The fit provides the value of $\tau_{\text {th }}=0.2 \mu \mathrm{s}$, which is also in agreement with independent FEM simulations.

\section{APPENDIX C: PHASE NOISE OF GAAS DISK OPTOMECHANICAL SELF-OSCILLATORS}

To complete informations given in the body of the text,

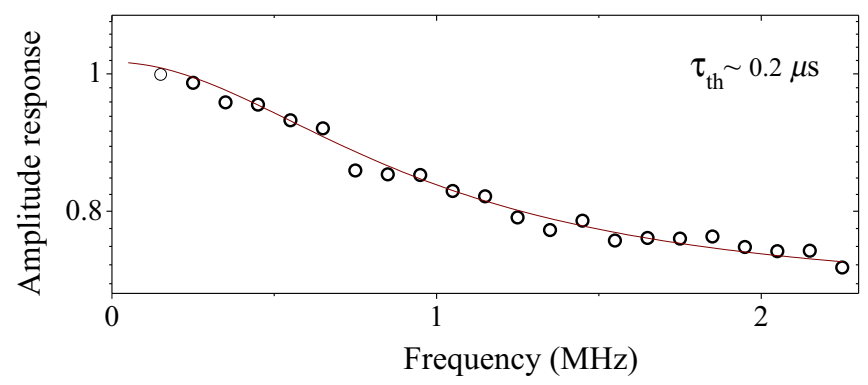

FIG. 7. Normalized frequency response of a GaAs disk optically pumped by a laser whose intensity is sinusoidally modulated. 

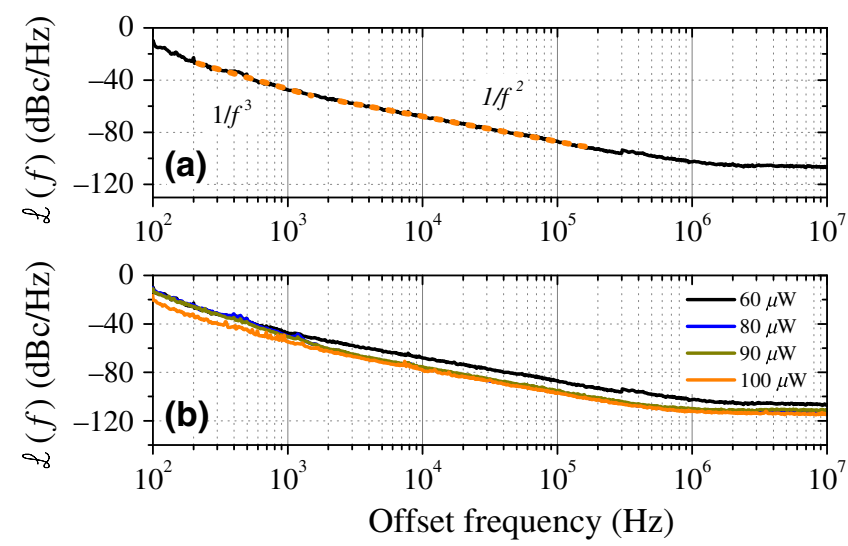

FIG. 8. (a) Phase-noise spectrum of a GaAs disk optomechanical self-oscillator integrated on the chip with a suspended GaAs taper waveguide. The dashed orange lines indicate the $1 / f^{3}$ and $1 / f^{2}$ dependences. (b) Phase-noise spectra of the same disk for varying optical drive power. The quoted power is measured at the output of the employed taper waveguide.

we show in Fig. 8 phase-noise spectra acquired on a selfoscillating GaAs disk fully integrated on the chip, with a suspended GaAs waveguide replacing the fiber taper [55]. The measurements are carried on a disk of radius $1.5 \mu \mathrm{m}$ and thickness $320 \mathrm{~nm}$. We observe in Fig. 8(a) that close to the carrier the phase noise varies as $1 / f^{3}$, while it recovers a $1 / f^{2}$ dependence at higher frequency offsets. The $1 / f^{3}$ dependence is a signature of "flicker noise" (alternatively known as "pink noise"), while the $1 / f^{2}$ regime still corresponds to the "white-noise" component [41]. Unlike the fiber-coupled disks shown in the main text, we notice the absence of spectral 'bumps' below $2 \times 10^{4} \mathrm{~Hz}$, probably as a consequence of more stable evanescent coupling conditions enabled by the on-chip waveguide. In Fig. 8(b), we observe again that the phase noise improves at higher drive power.

\section{APPENDIX D: FORCE GRADIENTS RESOLVED BY THE VERY STIFF PROBE}

We show in Fig. 9, in correspondance with Fig. 5, the external force gradient $\Delta k=\nabla F$ measured by the probe. In the optomechanical self-oscillation regime, we observe that an external force gradient as small as $(\Delta k \sim 0.9 \times$ $10^{-1} \mathrm{~N} / \mathrm{m}$ ) can be resolved. While this value is usual for a low-frequency cantilever type of resonator, it is unusual for a stiff $\left(k \sim 10^{5} \mathrm{~N} / \mathrm{m}\right)$ and ultra-high-frequency resonator of the kind explored here. The minimum detectable force variation can be estimated through the relation $F_{\min }=$ $A \times \nabla F_{\min }$, with $A$ the amplitude of the oscillatory motion. We inferred an amplitude of oscillation of $7 \mathrm{pm}$, leading to $F_{\min }=6 \times 10^{-13} \mathrm{~N}$, which comes close to the amplitude of Langevin forces in the measurement bandwidth

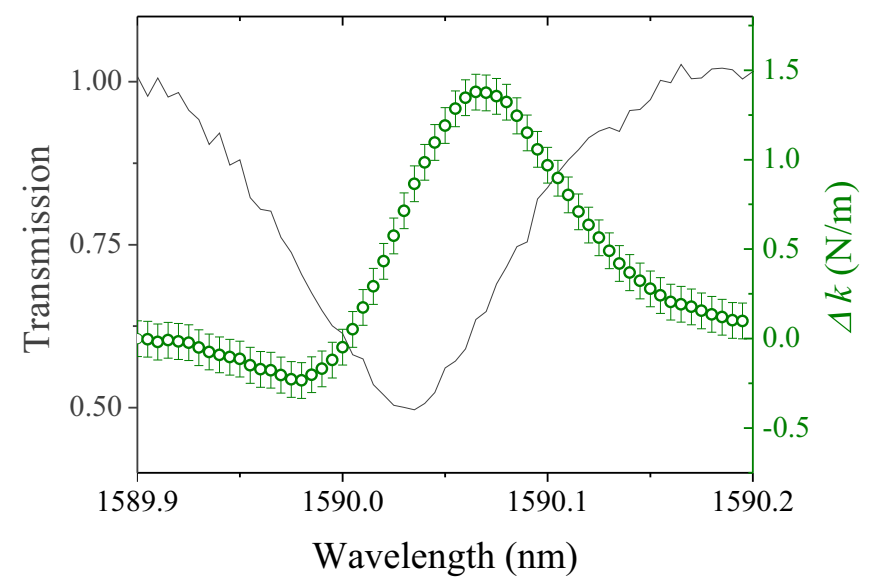

FIG. 9. Force sensing with the optomechanical self-oscillator. The force gradient $\Delta k$ sensed by the self-oscillator (green open circles) is shown as a function of the wavelength of the second laser (see text). The black curve represents the optical fiber taper transmission close to the resonance of WGM 2, acquired by time integrating the optical intensity in the second output channel when the disk is self-oscillating.

$B=300 \mathrm{~Hz}\left(4.7 \times 10^{-13} \mathrm{~N}\right)$, showing that the force sensor is approaching its thermodynamical limit.

In Fig. 10, we plot the photon number in WGM2 at equilibrium, after thermal relaxation, as a function of the wavelength $\lambda_{2}$ of the second laser. The asymmetry of the curve results from the thermo-optic effect, and is modeled here with the parameters of the above experiment and the mechanical resonator at rest, following a model employed in Ref. [31]. The thermo-optic effects is at the root of the asymmetry of the frequency shift observed in Fig. 5, as is

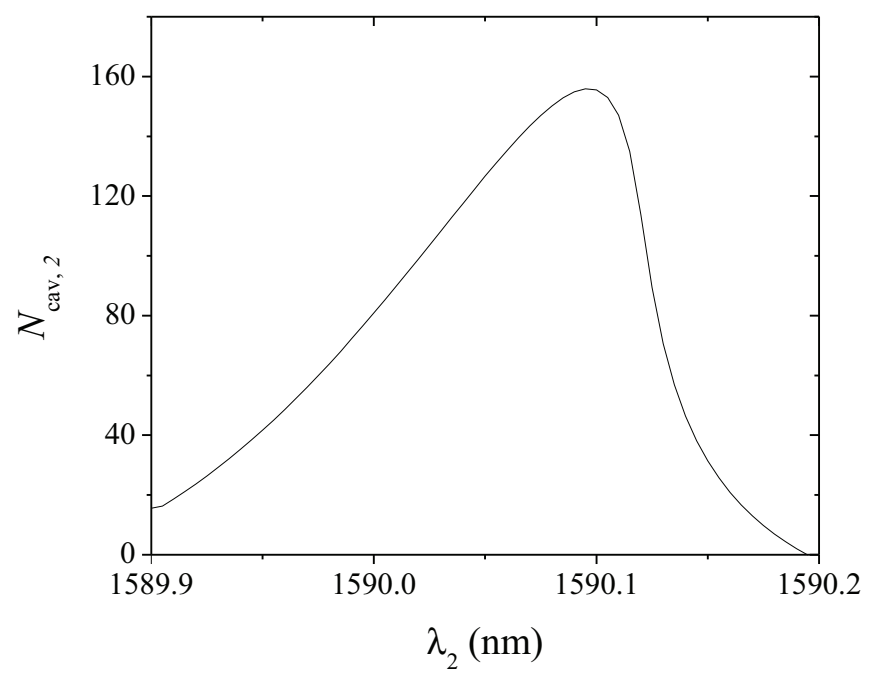

FIG. 10. Photon number in WGM2 as a function of the wavelength of the second laser, evaluated at equilibrium after thermal relaxation. 
already apparent in Eq. (4), which is linear in the equilibrium photon number. The asymmetry of the frequency shift can also be understood within the delayed-force model of optomechanics, which describes linear optomechanical feedback effects such as dynamical back action and the optical spring by subjecting the mechanical oscillator to a time-delayed force [34,56,57]. In the limit where $\kappa \gg\left(\omega_{m}, 1 / \tau_{\mathrm{th}}\right)$, which is valid here, this delayed-force model recovers the results obtained from the linearization of coupled equations Eq. (1) [24]. The model predicts a spring frequency shift proportional to the derivative of the steady-state force (at equilibrium) with respect to the mechanical displacement [34]. In the situation considered here this implies a frequency shift proportional to the derivative of the intracavity photon number plotted in Fig. 10 with respect to $\lambda_{2}$. The force gradient experienced by the mechanical oscillator in our experiments is hence asymmetric in the wavelength $\lambda_{2}$. Note, however, that integrated on a time scale $\gg\left(1 / \omega_{m}, \tau_{\text {th }}\right)$, the optical output response recovers in contrast a symmetric profile (black curve in Fig. 9). This feature is reproduced when directly solving the set of nonlinear equations Eq. (1) with an oscillatory motion $x(t)$ as input.

[1] T. Thundat, E. Wachter, S. Sharp, and R. Warmack, Detection of mercury vapor using resonating microcantilevers, Appl. Phys. Lett. 66, 1695 (1995).

[2] J. Tamayo, P. M. Kosaka, J. J. Ruz, Á. San Paulo, and M. Calleja, Biosensors based on nanomechanical systems, Chem. Soc. Rev. 42, 1287 (2013).

[3] N. V. Lavrik, M. J. Sepaniak, and P. G. Datskos, Cantilever transducers as a platform for chemical and biological sensors, Rev. Sci. Instrum. 75, 2229 (2004).

[4] A. Boisen, S. Dohn, S. S. Keller, S. Schmid, and M. Tenje, Cantilever-like micromechanical sensors, Rep. Prog. Phys. 74, 036101 (2011).

[5] N. V. Lavrik and P. G. Datskos, Femtogram mass detection using photothermally actuated nanomechanical resonators, Appl. Phys. Lett. 82, 2697 (2003).

[6] A. Cagliani and Z. J. Davis, Ultrasensitive bulk disk microresonator-based sensor for distributed mass sensing, J. Micromech. Microeng. 21, 045016 (2011).

[7] K. Ekinci, X. Huang, and M. Roukes, Ultrasensitive nanoelectromechanical mass detection, Appl. Phys. Lett. 84, 4469 (2004).

[8] Y. Yang, C. Callegari, X. Feng, K. Ekinci, and M. Roukes, Zeptogram-scale nanomechanical mass sensing, Nano Lett. 6, 583 (2006).

[9] J. Chaste, A. Eichler, J. Moser, G. Ceballos, R. Rurali, and A. Bachtold, A nanomechanical mass sensor with yoctogram resolution, Nat. Nanotechnol. 7, 301 (2012).

[10] I. Favero and K. Karrai, Optomechanics of deformable optical cavities, Nat. Photonics 3, 201 (2009).

[11] M. Aspelmeyer, T. J. Kippenberg, and F. Marquardt, Cavity optomechanics, Rev. Mod. Phys. 86, 1391 (2014).
[12] M. Li, W. H. P. Pernice, and H. X. Tang, Broadband allphotonic transduction of nanocantilevers, Nat. Nanotechnol. 4, 377 (2009).

[13] S. Forstner, S. Prams, J. Knittel, E. D. van Ooijen, J. D. Swaim, G. I. Haris, A. Szorkovszky, and B. W. P. H. Rubinsztein-Dunlop, Cavity Optomechanical Magnetometer, Phys. Rev. Lett. 108, 120801 (2012).

[14] F. Liu, S. Alaie, Z. Leseman, and M. Hossein-Zadeh, Subpg mass sensing and measurement with an optomechanical oscillator, Opt. Express 21, 19555 (2013).

[15] W. Yu, W. Jiang, Q. Lin, and T. Lu, Cavity optomechanical spring sensing of single molecules, Nat. Commun. 7, 12311 (2016).

[16] E. Gil-Santos, C. Baker, D. T. Nguyen, A. Lemaître, C. Gomez, S. Ducci, G. Leo, and I. Favero, High frequency nano-optomechanical disk resonators in liquids, Nat. Nanotechnol. 10, 810 (2015).

[17] K. Ekinci, Y. Yang, and M. Roukes, Ultimate limits to inertial mass sensing based upon nanoelectromechanical systems, J. Appl. Phys. 95, 2682 (2004).

[18] C. Höhberger and K. Karrai, in Proceedings of the 4th IEEE Conference on Nanotechnology (Munich, 2004), p. 419.

[19] C. Metzger Höhberger and K. Karrai, Cavity cooling of a microlever, Nature 432, 1002 (2004).

[20] T. Carmon, H. Rokhsari, L. Yang, T. J. Kippenberg, and K. J. Vahala, Temporal Behavior of Radiation-PressureInduced Vibrations of an Optical Microcavity Phonon Mode, Phys. Rev. Lett. 94, 223902 (2005).

[21] H. Rokhsari, T. J. Kippenberg, T. Carmon, and K. J. Vahala, Radiation-pressure-driven micro-mechanical oscillator, Opt. Express 13, 5293 (2005).

[22] O. Arcizet, P. F. Cohadon, T. Briant, M. Pinard, and A. Heidmann, Radiation pressure cooling and optomechanical instability of a micromirror, Nature 444, 71 (2006).

[23] C. Metzger, M. Ludwig, C. Neuenhahn, A. Ortlieb, I. Favero, K. Karrai, and F. Marquardt, Self-Induced Oscillations in an Optomechanical System Driven by Bolometric Backaction, Phys. Rev. Lett. 101, 133903 (2008).

[24] B. Guha, S. Mariani, A. Lemaître, S. Combrié, G. Leo, and I. Favero, High frequency optomechanical disk resonators in III-V ternary semiconductors, Opt. Express 25, 24639 (2017)

[25] T. R. Albrecht, P. Grütter, D. Horne, and D. Rugar, Frequency modulation detection using highq cantilevers for enhanced force microscope sensitivity, J. Appl. Phys. 69, 668 (1991).

[26] L. Ding, C. Baker, P. Senellart, A. Lemaître, S. Ducci, G. Leo, and I. Favero, High Frequency Gaas NanoOptomechanical Disk Resonator, Phys. Rev. Lett. 105, 263903 (2010).

[27] L. Ding, C. Baker, P. Senellart, A. Lemaître, S. Ducci, G. Leo, and I. Favero, Wavelength-sized gaas optomechanical resonators with gigahertz frequency, Appl. Phys. Lett. 98, 113108 (2011).

[28] L. Ding, C. Baker, A. Alessio, P. David, P. Senellart, A. Lemaître, S. Ducci, G. Leo, and I. Favero, Handbook of Optical Microcavities (PanStanford, Singapore, 2014), Chap. GaAs disk optomechanical resonators, p. 381.

[29] L. Ding, P. Senellart, A. Lemaître, S. Ducci, G. Leo, and I. Favero, Gaas micro-nanodisks probed by a looped fiber 
taper for optomechanics applications, Proc. SPIE 7712, 771211 (2010).

[30] C. Baker, W. Hease, D.-T. Nguyen, A. Andronico, S. Ducci, G. Leo, and I. Favero, Photoelastic coupling in gallium arsenide optomechanical disk resonators, Opt. Express 22, 14072 (2014).

[31] D. Parrain, C. Baker, G. Wang, B. Guha, E. G. Santos, A. Lemaître, P. Senellart, G. Leo, S. Ducci, and I. Favero, Origin of optical losses in gallium arsenide disk whispering gallery resonators, Opt. Express 23, 19656 (2015).

[32] E. Weidner, S. Combrié, A. de Rossi, N.-V.-Q. Tran, and S. Cassette, Nonlinear and bistable behavior of an ultrahighQ GaAs photonic crystal nanocavity, Appl. Phys. Lett. 90, 101118 (2007).

[33] V. R. Almeida and M. Lipson, Optical bistability on a silicon chip, Opt. Lett. 29, 2387 (2004).

[34] C. Metzger, I. Favero, A. Ortlieb, and K. Karrai, Optical self cooling of a deformable Fabry-Perot cavity in the classical limit, Phys. Rev. B 78, 035309 (2008).

[35] D. Parrain, T. Verdier, P. Senellart, A. Lemaître, S. Ducci, G. Leo, and I. Favero, Damping of optomechanical disks resonators vibrating in air, Appl. Phys. Lett. 100, 24 (2012).

[36] D. T. Nguyen, C. Baker, W. Hease, S. Sejil, P. Senellart, A. Lemaître, G. Leo, and I. Favero, Ultrahigh q-frequency product for optomechanical disk resonators with a mechanical shield, Appl. Phys. Lett. 103, 241112 (2013).

[37] D. T. Nguyen, W. Hease, C. Baker, E. Gil-Santos, P. Senellart, A. Lemaître, S. Ducci, G. Leo, and I. Favero, Improved optomechanical disk resonator sitting on a pedestal mechanical shield, New J. Phys. 17, 023016 (2015).

[38] M. Hamoumi, P. A. Allain, W. Hease, E. Gil-Santos, L. Morgenroth, B. Gérard, A. Lemaître, G. Leo, and I. Favero, Microscopic Nanomechanical Dissipation in Gallium Arsenide Resonators, Phys. Rev. Lett. 120, 223601 (2018).

[39] M. Hossein-Zadeh, H. Rokhsari, A. Hajimiri, and K. J. Vahala, Characterization of a radiation-pressure-driven micromechanical oscillator, Phys. Rev. A 74, 023813 (2006).

[40] A. L. Schawlow and C. H. Townes, Infrared and optical masers, Phys. Rev. 112, 1940 (1958).

[41] E. Rubiola, Phase Noise and Frequency Stability in Oscillators (Cambridge University Press, Cambridge, 2008).

[42] S. Tallur, S. Sridaran, and S. A. Bhave, A monolithic radiation-pressure driven low phase noise silicon nitride opto-mechanical oscillator, Opt. Express 19, 24522 (2011).

[43] A. Andronico, X. Caillet, I. Favero, S. Ducci, V. Berger, and G. Leo, Semiconductor microcavities for enhanced non- linear optics interactions, J. Eur. Opt. Soc.-Rapid Publ. 3, 08030 (2008).

[44] W. P. Jakubik, Surface acoustic wave-based gas sensors, Thin Film Solids 520, 986 (2011).

[45] S. Fanget, S. Hentz, P. Pugeta, J. Arcamone, M. Matheron, E. Colinet, P. Andreucci, L. Duraffourg, E. Myers, and M. L. Roukes, Gas sensors based on gravimetric detection, a review, Sens. Actuators B: Chem. 160, 804 (2011).

[46] A. Vinante, M. Bonaldi, F. Marin, and J. Zendri, Dissipative feedback does not improve the optimal resolution of incoherent force detection, Nat. Nanotechnol. 8, 470 (2013).

[47] G. I. Harris, D. L. McAuslan, T. M. Stace, A. C. Doherty, and W. P. Bowen, Minimum Requirements for Feedback Enhanced Force Sensing, Phys. Rev. Lett. 111, 103603 (2013).

[48] J. Philippe, G. Arndt, E. Colinet, M. Savoye, T. Ernst, E. Ollier, and J. Arcamone, in Proceedings of IEEE MEMS (San Francisco, 2014), p. 1071.

[49] G. Gourlat, M. Sansa, G. Jourdan, P. Villard, G. Sicard, and S. Hentz, in Proceedings of IEEE International Frequency Control Symposium (Denver, 2015).

[50] M. Sansa, G. Gourlat, G. Jourdan, M. Gely, P. Villard, G. Sicard, and S. Hentz, Compact heterodyne nems oscillator for sensing applications, Solid State Electron. 125, 214 (2016).

[51] D. Y. Abramovitch, in Proceedings of the 2011 American Control Conference (San Francisco, 2011).

[52] D. Y. Abramovitch, in Proceedings of the 18th World Congress of The International Federation of Automatic Control Vol. 44 (Milano, 2011), p. 12721.

[53] M. Ludwig, B. Kubala, and F. Marquardt, The optomechanical instability in the quantum regime, New J. Phys. 10, 095013 (2008).

[54] J. Qian, A. Clerk, K. Hammerer, and F. Marquardt, Quantum signatures of the optomechanical instability, New J. Phys. 109, 253601 (2012).

[55] C. Baker, A. Andronico, S. P A. Lemaître, E. Galopin, S. Ducci, G. Leo, and I. Favero, Critical optical coupling between a gaas disk and a nanowaveguide suspended on the chip, Appl. Phys. Lett. 10, 151117 (2011).

[56] J. Restrepo, J. Gabelli, C. Ciuti, and I. Favero, Classical and quantum theory of photothermal cavity cooling of a mechanical oscillator, C. R. Phys. 12, 860 (2011).

[57] P. F. Cohadon, J. Harris, F. Marquardt, and L. Cugliandolo, eds., Quantum Optomechanics and Nanomechanics, Lecture Notes of the Les Houches Summer School, August 2015 (Oxford University Press, Cambridge, 2020), Chap. Optomechanical Interactions. 